\title{
Cerebellar Liponeurocytoma Presenting with Fatal Tumor Hemorrhage
}

\section{Elia Guadagno, $M D^{1}$, Lorenzo Ugga, $M D^{2}$, Annarita Gencarelli, $M D^{1}$, Valentina Orlando, MD³, Sergio Corvino, $M D^{3}$, Maria Rosaria Cervasio, $M D^{1}$, Sara Pignatiello, $M D^{1}$, Andrea Elefante, $M D^{2}$, Francesco Maiuri, $M^{3^{*}}$ and Maria Laura Del Basso De Caro, MD $^{1}$}

\author{
${ }^{1}$ Department of Advanced Biomorphological Sciences, Section of Pathology, "Federico II" University of \\ Naples, Naples, Italy \\ ${ }^{2}$ Department of Advanced Biomorphological Sciences, Section of Radiology, "Federico II" University of \\ Naples, Naples, Italy \\ ${ }^{3}$ Department of Neurosciences and Reproductive and Odontostomatological Sciences, Neurosurgical \\ Clinic, "Federico II" University School of Medicine, Naples, Italy
}

*Corresponding author: Francesco Maiuri, MD, Department of Neurosciences and Reproductive and Odontostomatological Sciences, Neurosurgical Clinic, "Federico II" University School of Medicine, Via Pansini, 5, 80131, Napoli, Italy, Tel: +39-81-7462581, Fax: +39-81-7462594

\begin{abstract}
Liponeurocytoma is a very rare tumor of the nervous system, with only 63 reported cases, mainly in the cerebellum and ventricular system. The key-points for the diagnosis are the histological findings of neurocytic cells with lipidic accumulation, with positivity for S-100 and synaptophysin and negativity for IDH-1, and the MR finding of hyperintensity areas on T1Wi corresponding to fatty tissue within the tumor.

This article reports a case of cerebellar liponeurocytoma presenting with acute ictal onset due to significant tumor hemorrhage and resulting in poor outcome with death, after urgent surgery with complete tumor resection. Inspite of the absence of fatty areas on MRI due to the blood infiltration, limited areas of fatty accumulation in the neurocytic tumor cells were evidenced on the deep histological sections, thus allowing the correct diagnosis.
\end{abstract}

\section{Keywords}

Cerebellar liponeurocytoma, Tumor hemorrhage, Magnetic resonance, Immunohistochemistry

\section{Introduction}

Cerebellar liponeurocytoma is a very rare neoplasm of the adults, first described by Bechtel, et al. in 1978 [1], as mixed mesenchymal and neuroectodermal tu- mor. The term "liponeurocytoma" was first introduced in the 2000 World Health Organization as "a distinct clinico-pathological entity in the group of glioneuronal tumors" [2]. Now, it is defined as a WHO grade II tumor with neuronal consistent, variable astrocytic and focal lipomatous differentiation and with infiltrative nature, low proliferation index, but high likelihood of recurrence [3-4].

This article reports a rare case of cerebellar liponeurocytoma presenting with fatal tumor hemorrhage and discusses the differential pathological diagnosis.

\section{Case Description}

\section{Clinical history and diagnosis}

A 49-years-old woman had a history of arterial hypertension and chronic renal insufficiency treated by failed kidney transplant one month before and dialysis since 2011; besides, she was in therapy with clopidogrel and acetylsalycilic acid because of deep arterial thrombosis.

In January 2018, the patient had intense headache,

Citation: Guadagno E, Ugga L, Gencarelli A, Orlando V, Corvino S, et al. (2019) Cerebellar Liponeurocytoma Presenting with Fatal Tumor Hemorrhage. Neurosurg Cases Rev 2:026. doi.org/10.23937/26434474/1710026

Accepted: October 16, 2019; Published: October 18, 2019

Copyright: (C) 2019 Guadagno E, et al. This is an open-access article distributed under the terms of the Creative Commons Attribution License, which permits unrestricted use, distribution, and reproduction in any medium, provided the original author and source are credited. 


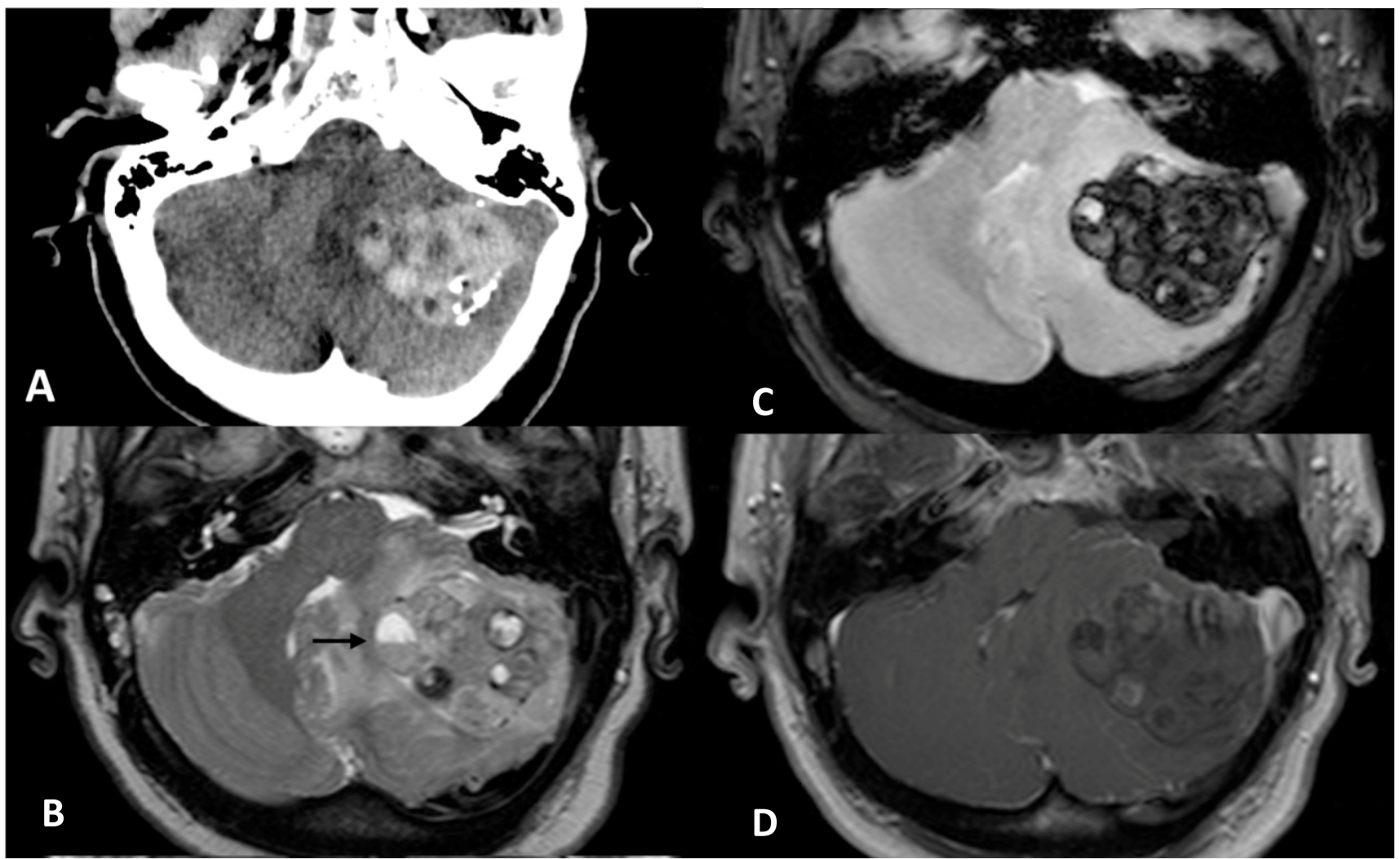

Figure 1: a) Computerized Tomography of the skull showing a left cerebellar hemorrhage with small intralesional laminar calcifications; b-d) Magnetic Resonance images depict a left cerebellar multiloculated tumor lesion compressing the brainstem and displacing the $4^{\text {th }}$ ventricle. On T2 and T1Wl; b,c) The lesion shows some blood-fluid levels (black arrow), inhomogeneous signal due to blood products in different stages, and vasogenic edema; d) No areas with fat signal are present. The tumor does not enhance after contrast administration.

vomiting and rapidly progressive deterioration of the consciousness. An urgent computerized tomography (CT) (Figure 1a) at our hospital revealed a $42 \mathrm{~mm}$ hematoma of the left cerebellar hemisphere, with calcifications and perilesional edema, causing compression of the brainstem and triventricular hydrocephalus. Thus, an external ventricular drainage was immediately placed. Magnetic resonance imaging (MRI) (Figure $1 \mathrm{~b}$, Figure $1 \mathrm{c}$ and Figure $1 \mathrm{~d}$ ) showed a tumor mass of the left cerebellar hemisphere $(52 \times 33 \times 27 \mathrm{~mm})$ with heterogeneous signal due to the presence of peripheral calcifications, diffuse intralesional bleeding, solid nodules and cystic and necrotic components; discrete perilesional edema, severe brainstem compression, triventricular hydrocephalus and herniation of the left cerebellar tonsil were present. DWI, spectroscopy and perfusion sequences did not provide further information because of the diffuse intralesional blood.

At admission the patient was comatose and with equal and reactive pupils, then she rapidly deteriorated up to GCS 5 and was recovered in our intensive care unit and intubated.

\section{Surgery and outcome}

An urgent midline and left suboccipital craniectomy associated to $\mathrm{C} 1$ laminectomy was performed. After left cerebellar corticotomy a large reddish tumor mass was exposed; it had slightly increased consistency, vascularized soft tissue, significant blood collection and necrosis. Piecemeal tumor resection was realized. Significant brainstem displacement was evident. The edematous left cerebellar tonsil, displaced downward into the occipital foramen, was resected. The intraoperative diagnosis on frozen sections was low grade glioma. After surgery the patient remained intubated and under pharmacological sedation, with enlarged and scarcely reactive pupils.

In the first postoperative day a CT scan revealed a blood collection in the surgical cavity, enlarged ventricular system and edema of the pons and medium cerebellar peduncle. Thus, the patient was re-operated on and the blood clot was evacuated from the surgical cavity. In the third postoperative day fixed not reactive mydriasis and no motor response were evident (GCS 3); the hemodynamic parameters were instable despite the high doses of noradrenaline. The patient died for cardiac arrest.

\section{Pathology}

Pathological examination of the first H-E sections revealed a tumor with mixed patterns, including areas with tortuous and variably dilated vessels with marked hyalinization (Figure 2a) and areas with moderate cellularity (Figure 2b) composed by cells with clear cyto- 


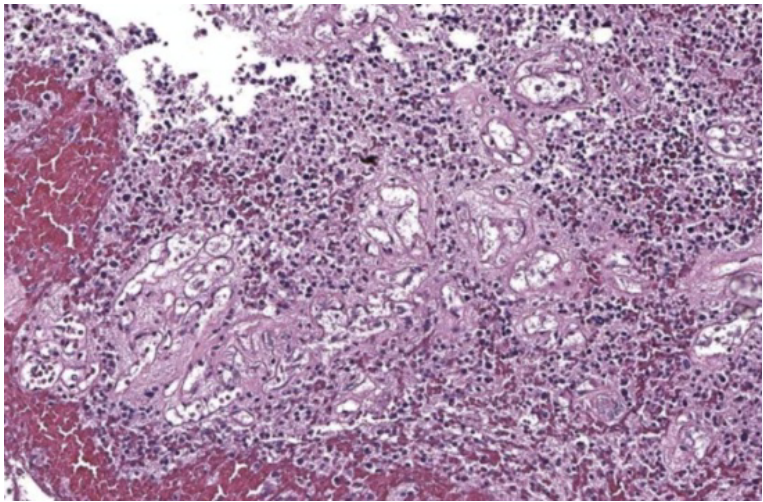

a

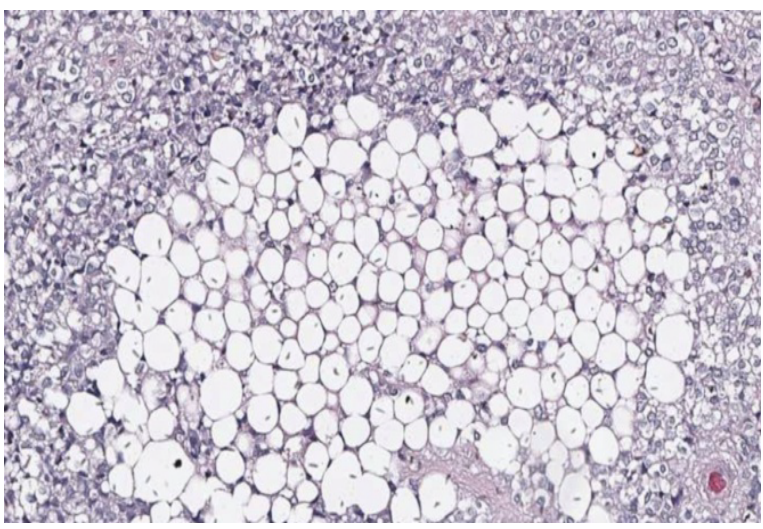

c

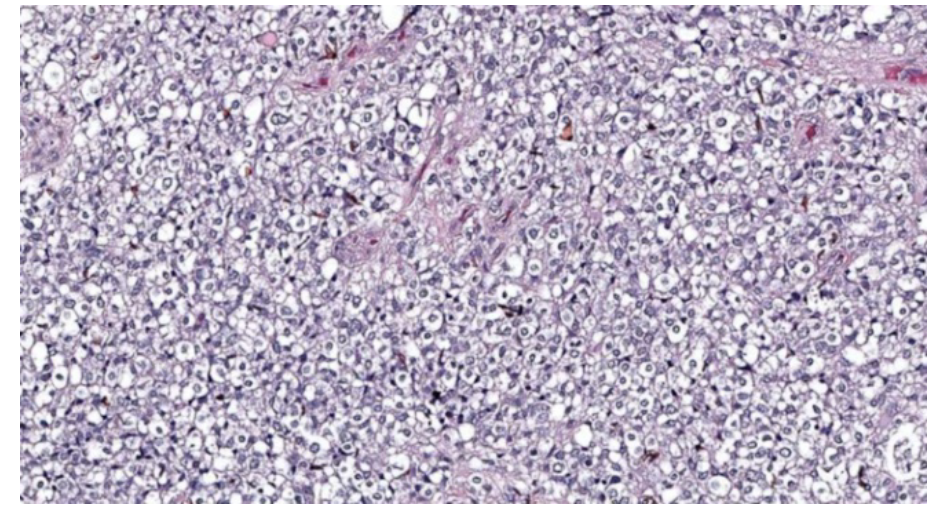

b

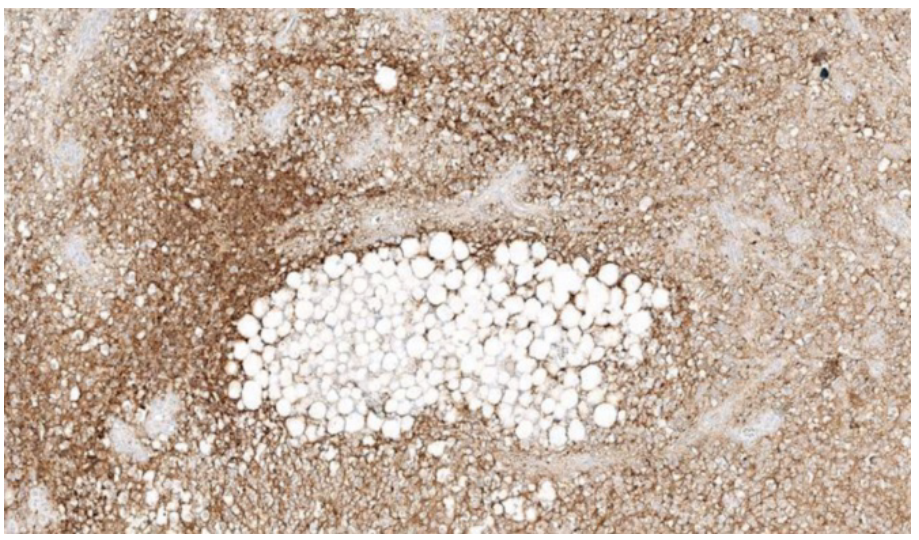

d

Figure 2: a) Tumor characterized by epithelioid clear to foamy stromal cells mixed with small vessels with hyalinized walls (H-E stain, 200× magnification); b) Areas with moderate cellularity, composed by elements with cytoplasm clearing and a central nucleus (H-E stain, 200× magnification); c) A deep section highlighted focal accumulation of lipid-laden cells (H-E stain, 100x magnification); d) Immunoreactivity for synaptophysin protein.

plasm and central spherical nucleus interspersed with a delicate capillary network with "chicken-like" appearance. Microcalcifications were also evident. Some eosinophilic granular bodies were observed within the perilesional parenchyma.

The examination of the deep sections revealed the presence of focal accumulation of lipid-laden cells (Figure 2c), consisting of neuroepithelial tumor cells with lipidic accumulation.

The immunohistological studies revealed diffuse reactivity for S-100 and synaptophysin (Figure 2d) and focal for GFAP. Cytokeratin and EMA were negative, thus excluding a cellular variant of hemangioblastoma. The IDH 1 was negative, thus excluding an oligodendroglioma. The p53 was expressed in less than $10 \%$ of the neoplastic cells. The Ki63 Li was 2-3\%.

The histological and immunohistochemical findings allowed the diagnosis of liponeurocytoma.

\section{Discussion}

This case is notewhorthy for the exceptional occurrence of this tumor entity and the sudden clinical onset with severe hemorrhage.

Liponeurocytoma of the central nervous system is very unusual, with 47 reported cases in the cerebellum
[5-8], 14 in the supratentorial ventricular system $[9,10]$, one in the cerebellopontine angle [11] and one in the cerebral parenchyma [12], as evidenced by some literature reviews [10,12-16]. We agree with Cai et al. [12] that the definition of "central liponeurocytoma" is more appropriate than "cerebellar liponeurocytoma" for including the different reported sites.

Almost all reported cases were observed in patients from 30 to 60-years-old, with no sex difference. The clinical presentation was aspecific and consistent with a slowing growing mass lesion.

In our cases the clinical onset was that of a severe cerebellar hemorrhage with rapidly progressive neurological deterioration, coma and signs of severe brainstem compression requiring respiratory assistance, in absence of any previous neurological symptom. The hemorrhage within a moderately vascularized tumor was favored by several conditions, including chronic hypertension, dialysis and therapy with clopidogrel and acetyl salycilic acid. Also, re-bleeding occurred inspite of the complete tumor resection. Only one among the reported cases of liponeurocytoma [5] was associated to hemorrhage; however, the clinical evolution was not acute but progressive and the bleeding was evidenced only on MRI. 
The MRI appearance of liponeurocytoma is that of a tumor mass of inhomogeneous signal intensity, with isointense areas in both T1W1 and T2Wi, due to high cellular density, hyperintense cysts and spots of hypointensity that indicate areas of calcifications. The presence of focal areas of hyperintensity on T1W1 corresponding to fatty tissue inside the tumor is a characteristic finding found in almost all reported cases and may suggest the preoperative diagnosis of liponeurocytoma $[10,12,17]$. However, in our own, as in another reported case [12], no areas with fat signal intensity were detected. This may be due to both the scarce amount of tumor cells with lipidic accumulation and the hemorrhage infiltration. The contrast enhancement is slightly inhomogeneous implying mild vascularity [16].

The typical histological aspect of liponeurocytoma is that of a tumor with mixed pattern, with areas characterized by dilated vessels and hyalinization, and areas with moderate cellularity, composed by neurocytic cells with focal lipomatous degeneration. This last findings should suggest the diagnosis of liponeurocytoma, in spite of the exceptional occurrence of this pathological entity.

The differential diagnosis must include other more frequent neoplasms with possible lipomatous degeneration, such as oligodendroglioma, hemangioblastoma, neurocytoma, medulloblastoma. The immunohistochemical studies are useful to this aim.
The immunohistochemical profile of liponeurocytoma reveals both neuronal and glial differentiation, with positivity for synaptophysin, NSE and MAP-2, focal expression of GFAP and positivity to TP53 (20\%). Oligodendroglioma is negative for neuronal markers, such as synaptophysin, and shows high frequency of IDH1 mutation. The cellular variant of the hemangioblastoma, a tumor with extremely variable cell density, dilated vessels and cystic-like spaces, may be positive to inhibin, is negative for GFAP and TP53 and only occasionally shows lipomatous differentiation. The medulloblastoma shows the typical nodular architecture, high cellularity and MIB-1 index, absence of adypocite-like (vacuolated) tumor cells. A diagnostic algorithm of these immunohistochemical studies may be proposed to aid the differential diagnosis of clear cell cerebellar tumors (Figure 3 ).

Total surgical resection is the treatment of choice of liponeurocytoma. Radiation therapy may be considered for tumors with high proliferation index and for local recurrences.

Although the prognosis is favorable for the more benign forms, the reported recurrence rate is $31 \%[17,18]$ and cases with aggressive pathological and clinical behaviour are described $[18,19]$. However, the guidelines of adjuvant treatments, the progression-free survival and the prognosis are difficult to be exactly defined, because of the exceptional occurrence of this tumor, the scarce knowledge of its natural history and the lack of

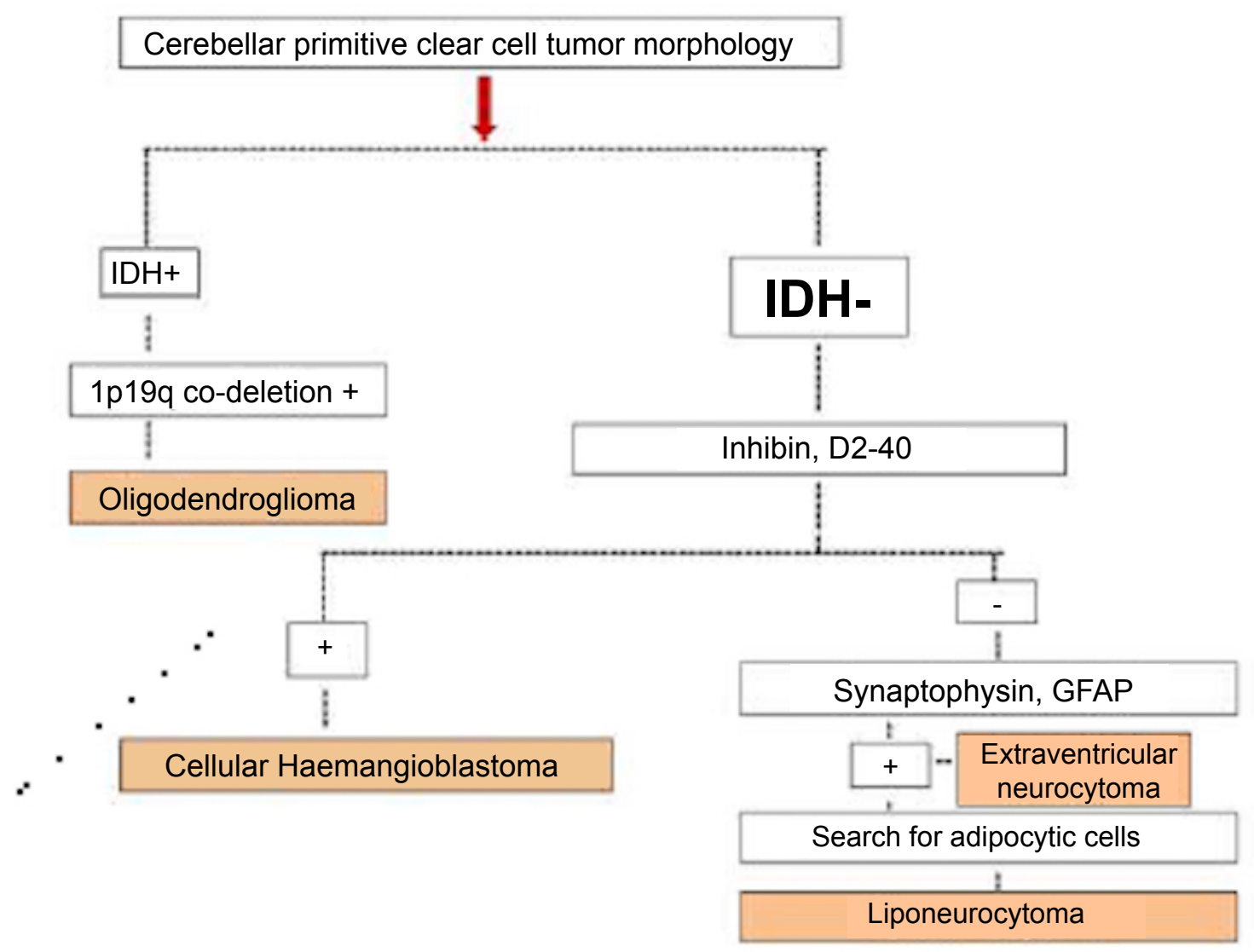

Figure 3: Proposal of diagnostic algorithm useful in differentiating clear cell cerebellar tumor lesions. 
follow-up data.

\section{Conclusion}

Liponeurocytoma is a very rare tumor of the cerebellum and ventricular system, which may be diagnosed from the histological finding of neurocytic cells with focal lipidic accumulation and hyperintensity on T1WI of MR corresponding to fatty tissue within the tumor. Even when this radiological finding is not evident, as in our case with fatal tumor hemorrhage, the careful investigation of small focal areas of lipidic accumulation is mandatory to define diagnosis.

\section{References}

1. Bechtel JT, Patton JM, Takei Y (1978) Mixed mesenchymal and neuroectodermal tumor of the cerebellum. Acta Neuropathol 41: 261-263.

2. Kleihues P, Chimelli L, Giangaspero F (2000) Cerebellar liponeurocytoma. In: Kleihues P, Cavenee WK, Pathology and genetics of tumours of the nervous system. IARC Press, Lyon, 110-111.

3. Kleihues $P$, Chimelli L, Giangaspero F, Louis DN, Ohgaki $\mathrm{H}$, et al. (2015) Cerebellar liponeurocytoma. World health organization classification of tumors of the central nervous system. IARC, Lyon, France, 110-112.

4. Louis DN, Perry A, Reifenberger G, von Deimling A, Figarella-Branger D, et al. (2016) The 2016 world health organization classification of tumors of the central nervous system: A summary. Acta Neuropathol 131: 803-820.

5. Jackson TR, Regine WF, Wilson D, Davis DG (2001) Cerebellar liponeurocytoma: Case report and review of the literature. J of Neurosurg 95: 700-703.

6. Nishimoto T, Kaya B (2012) Cerebellar liponeurocytoma. Arch Pathol Lab Med 136: 965-969.

7. Oudrhiri MY, Raouzi N, El Kacemi I, N El Fatemi, R Gana, et al. (2014) Understanding cerebellar liponeurocytomas: Case report and literature review. Case Rep Neurol Med.

8. Wolf A, Alghefari H, Krivosheya D, Staudt, Bowden G, et al. (2015) Cerebellar liponeurocytoma: A rare intracranial tumor with possible familial predisposition. Case report. J Neurosurg 1-5.

9. Kuchelmeister K, Nestler U, Siekmann R, Schachenmayr W (2006) Liponeurocytoma of the left lateral ventricle: Case report and review of the literature. Clin Neuropathol 25: 8694.

10. Chakraborti S, Mahadevan A, Govindan A, Yasha TC, Santosh V, et al. (2011) Supratentorial and cerebellar liponeurocytomas: Report of four cases with review of literature. $J$ Neurooncol 103: 121-127.

11. Chiaramonte $C$, Rabaste $S$, Jacquesson $T$, Meyronet $D$, Francois Cotton, et al. (2018) Liponeurocytoma of the cerebellopontine angle. World Neurosurg 112: 18-24.

12. Cai J, Li W, Du J, Xu N, Gao P, et al. (2018) Supratentorial intracerebral cerebellar liponeurocytoma. A case report and literature review. Medicine 97: 2 .

13. Anghileri E, Eoli M, Paterra R, Ferroli P, Pollo B, et al. (2012) FABP4 is a candidate marker of cerebellar liponeurocytomas. J Neurooncol 108: 513-519.

14. Karabagli P, Sav A, Pamir N (2014) Does "cerebellar liponeurocytoma" always reflect an expected site? An unusual case with a review of the literature. Folia Neuropathol 52: 101-105.

15. Kakkar A, Sable M, Suri V (2015) Unusual tumor of the central nervous system- ultrastructural examination. Ultrastruct Pathol 39: 419-423.

16. Xu N, Cai J, Du J, Yang R, Zhu H, et al. (2017) Clinical features and prognosis for intraventricular liponeurocytoma. Oncotarget 8: 62641-62647.

17. Limaiem F, Bellil S, Chelly I, Bellil K, Mekni A, et al. (2009) Recurrent cerebellar liponeurocytoma with supratentorial extension. Can J Neurol Sci 36: 662-665.

18. Chatillon CE, Guiot MC, Roberge D, Leblanc R (2009) Cerebellar liponeurocytoma with high proliferation index : Treatment options. Can J Neurol Sci 36: 658-661.

19. Jenkinson MD, Bosma JJD, Du Plessis D, Ohgaki H, Kleihues $P$, et al. (2003) Cerebellar liponeurocytoma with an unusually aggressive clinical course: Case report. Neurosurgery 53: 1425-1428. 